\section{OPEN ACCESS}

Edited by:

Dahua Yu,

Inner Mongolia University of Science and Technology, China

Reviewed by:

Shuang Xia

Tianjin First Central Hospital, China

Jun Jiang,

Shanghai Normal University, China

${ }^{*}$ Correspondence:

Yuxin Shi

shiyuxin@shphc.org.cn

Dan-Chao Cai

caidanchao@shphc.org.cn

tThese authors have contributed equally to this work

Specialty section:

This article was submitted to Applied Neuroimaging, a section of the journal

Frontiers in Neurology

Received: 13 August 2021 Accepted: 22 December 2021 Published: 14 January 2022

Citation:

Ma Q, Shi X, Chen G, Song F, Liu F, Zheng H, Shi Y and Cai D-C (2022) HIV-Associated Structural and Functional Brain Alterations in Homosexual Males.

Front. Neurol. 12:757374 doi: 10.3389/fneur.2021.757374

\title{
HIV-Associated Structural and Functional Brain Alterations in Homosexual Males
}

\section{Qiong $\mathrm{Ma}^{1,2+}$, Xiudong Shi ${ }^{2 t}$, Guochao Chen ${ }^{1,2}$, Fengxiang Song ${ }^{2}$, Fengjun Liu ${ }^{2}$, Huang Zheng ${ }^{3}$, Yuxin Shi ${ }^{2 *}$ and Dan-Chao Cai ${ }^{2 *}$}

${ }^{1}$ Shanghai Institute of Medical Imaging, Fudan University, Shanghai, China, ${ }^{2}$ Department of Radiology, Shanghai Public Health Clinical Center, Fudan University, Shanghai, China, ${ }^{3}$ Shanghai Commercial Sex Worker (CSW) \& Man Have Sex With Man (MSM) Center, Shanghai, China

Purpose: Neuroimaging elucidations have shown structural and functional brain alterations in HIV-infected (HIV+) individuals when compared to HIV-negative (HIV-) controls. However, HIV - groups used in previous studies were not specifically considered for sexual orientation, which also affects the brain structures and functions. The current study aimed to characterize the brain alterations associated with HIV infection while controlling for sexual orientation.

Methods: Forty-three HIV+ and $40 \mathrm{HIV}$ - homosexual men (HoM) were recruited and underwent resting-state MRI scanning. Group differences in gray matter volume (GMV) were assessed using a voxel-based morphometry analysis. Brain regions with the altered GMV in the HIV+ HoM group were then taken as regions of interest in a seedbased analysis to identify altered functional connectivity. Furthermore, the amplitude of low-frequency fluctuation (ALFF) and regional homogeneity values were compared between the two groups to evaluate the HIV-associated functional abnormalities in local brain regions.

Results: HIV+ HoM showed significantly increased GMV in the bilateral parahippocampal gyrus and amygdala, and decreased GMV in the right inferior cerebellum, compared with the HIV- HoM. The brain regions with increased GMV were hyper-connected with the left superior cerebellum, right lingual gyrus, and left precuneus in the HIV+ HoM. Moreover, the ALFF values of the right fusiform gyrus, and left parahippocampal gyrus were increased in the HIV+ HoM. The regional homogeneity values of the right anterior cingulate and paracingulate gyri, and left superior cerebellum were decreased in the HIV+ HoM.

Conclusion: When the study population was restricted to HoM, HIV+ individuals exhibited structural alterations in the limbic system and cerebellum, and functional abnormalities in the limbic, cerebellum, and visual network. These findings complement the existing knowledge on the HIV-associated neurocognitive impairment from the previous neuroimaging studies by controlling for the potential confounding factor, sexual orientation. Future studies on brain alternations with the exclusion of related factors like sexual orientation are needed to understand the impact of HIV infection on neurocognitive function more accurately.

Keywords: homosexual, HIV infection, gray matter volume, functional connectivity, amplitude of low frequency fluctuation, regional homogeneity 


\section{INTRODUCTION}

Even with the success of combination antiretroviral therapy (cART), various neurological complications caused by the infiltration of the human immunodeficiency virus (HIV) in the central nervous system (CNS) (1), especially HIV-associated neurocognitive disorder (2), remain a heavy disease burden. Neuroimaging is a vital tool to provide insight into structural, functional, and molecular changes occurring in the brain and has the potential to comprehensively elucidate the pathogenesis of HIV-associated neurocognitive disorder $(3,4)$. Structural magnetic resonance imaging (sMRI) studies of HIV-infected $(\mathrm{HIV}+)$ individuals have found widespread brain atrophy and volume reduction in the subcortical structures including caudate nucleus, putamen, amygdala, thalamus, hippocampus, and parahippocampus (5-8), and cerebellar $(9,10)$. Resting-state functional MRI (rs-fMRI) studies revealed that HIV+ individuals had certain attenuation in brain intra- and internetwork connections, especially in the default mode, salience, and executive control networks, which might underlie the reported cognitive impairments $(11,12)$. However, these studies were inconsistent in regional distribution and effect sizes of brain abnormalities, and their conclusions cannot be generalized to the whole HIV+ cohort.

The inconsistencies in neuroimaging studies of HIV infection might be caused by multiple confounding factors, including age, sex, and substance use. For instance, age and HIV synergistically deteriorate cognitive performance through overlapping pathogenic mechanisms (13). One study reported that age exacerbated HIV-associated white matter abnormalities and fronto-subcortical white matter integrity loss (14). Numerous neuroimaging studies also showed brain atrophy, white matter damage and functional network strength, as a result of substance use in persons living with HIV (4). Discrepant results from previous studies might also be due to the heterogeneity of clinical status in studied samples, such as cART initiation and adherence, route of HIV infection, stage of HIV infection, viral suppression, and CD4 cell counts. For example, HIV+ individuals with constant cART showed greater functional connectivity in fronto-striatal networks than HIV+ individuals without cART (15), which was possibly related to the reduced inflammatory response and glial activation during cART (16). To sum up, different confounding factors might have different effects on alterations in the structure and function of brain, which to some extent affects the understanding of HIV infection effects. And many other factors have not been taken into account.

Sexual orientation is a biological phenomenon for unknown reasons. Many previous studies have investigated the brain differences between differing sexual orientations after controlling for potential mediating factors including HIV infection and substance abuse. sMRI showed, compared with the heterosexual men $(\mathrm{HeM})$, the homosexual men (HoM) displayed thinner visual cortex and thicker patietal cortex, smaller thalamus and larger corpus callosum $(17-19,48)$. A positron emission tomography study that measured the cerebral blood flow showed that the amygdala connections in HoM were more widespread from the left amygdala whereas those in HeM were more widespread from the right amygdala (20). Furthermore, a series of rs-fMRI studies revealed differences in regional homogeneity, amplitude of low-frequency fluctuation (ALFF), and functional connectivity between HoM and HeM $(21,22)$. Sexual transmission is currently one of the main modes of HIV transmission. A recent investigation showed that gay men and other men who have sex with men accounted for an estimated $17 \%$ of new HIV infections globally (23). However, as a potential confounding factor, sexual orientation has not been specifically considered when investigating the effects of HIV infection on brain alteration.

Considering this background, we designed a prospective MRI study, which controls for the sexual orientation in addition to other confounding factors controlled in previous HIV neuroimaging studies, to investigate the structural and functional changes in the HIV+ individuals. To be more specific, we limited our samples to HIV+ or HIV- HoM. We collected their sMRI and rs-fMRI data, performed voxel-based morphometry (VBM) and seed-based functional connectivity analysis, and calculated the ALFF and regional homogeneity values. The purpose of the current study was to determine how HIV alters the brain structure and function when sexual orientation was explicitly controlled as the homosexual males.

\section{MATERIALS AND METHODS}

\section{Participants}

A total of 43 HIV+ HoM (age $28.23 \pm 3.80$ ranging from 21 to 35 years) and $40 \mathrm{HIV}-\mathrm{HoM}$ (age $27.80 \pm 4.49$ ranging from 19 to 35 years) were recruited in this current study. There was no significant group difference in the mean age $\left[t_{(81)}=\right.$ $-0.475, P=0.636, \mathrm{BF}_{+0}=0.25$, with median posterior $\delta=$ $-0.09,95 \% \mathrm{CI}=(-0.50,0.31)]$. The inclusion criteria were: (1) adult men aged between 18 and 35 years; (2) right-handed subjects; (3) individuals that were able to sign informed consent. The exclusion criteria were: (1) individuals with the history of confounding neurological diseases including multiple sclerosis, Parkinson's disease, epilepsy, or dementia; (2) individuals with current or past opportunistic central nervous system infection; (3) people with head injury with loss of consciousness longer than $30 \mathrm{~min}$; (4) individuals with the existence of psychiatric disorders including schizophrenia, depression or anxiety; (5) individuals having a history of alcohol or drug abuse; (6) individuals with MRI contraindication. The clinical assessment characteristics are presented in Table 1. Among the $43 \mathrm{HIV}+$ HoM, 28 received the standard neurocognitive tests before the MRI scan. None of the tested participants were diagnosed as cognitive impairment (global deficit score $\geq 0.5$ ) (24). This study was approved by the institutional review board of Shanghai Public Health Clinical Center, and all the participants provided written informed consents.

\section{MRI Data Acquisition}

Scanning was performed using an Ingenia 3.0 T scanner (Philips, Amsterdam, Netherlands) with an 8-channel phase-array head coil at the Shanghai Public Health Clinical Center. For the 
TABLE 1 | Demographics, clinical information, and neurocognitive performance of HIV+ HoM and HIV- HoM.

\begin{tabular}{|c|c|c|c|c|}
\hline Category & HIV+ HoM $(n=43)$ & HIV- HoM $(n=40)$ & $P$-value & BF \\
\hline Age (years) & $28.23 \pm 3.80$ & $27.80 \pm 4.49$ & 0.636 & 0.25 \\
\hline Duration of HIV infection (months) & $43.00(18.00-66.00)$ & $\dagger$ & & \\
\hline Duration of cART (months) & $39.00(17.00-57.00)$ & $\dagger$ & & \\
\hline Current CD4+ cell count (cells/ $\mu l)$ & $479.65 \pm 179.56(353.00-640.00)$ & $\dagger$ & & \\
\hline Nadir CD4+ cell count (cells/ $\mu$ l) & $247.86 \pm 117.76(183.00-310.00)$ & $\dagger$ & & \\
\hline Current CD8+ cell count (cells/ $\mu \mathrm{l})$ & $807.47 \pm 282.41(622.00-994.00)$ & $\dagger$ & & \\
\hline Highest CD8+ cell count (cells/ $\mu l)$ & $1,140.23 \pm 451.26(819.00-1,381.00)$ & $\dagger$ & & \\
\hline Current viral load & & $\dagger$ & & \\
\hline$<20, n(\%)$ & $9(21 \%)$ & $\dagger$ & & \\
\hline Not detected, $n(\%)$ & $31(72 \%)$ & $\dagger$ & & \\
\hline GDS, $n$ & $0.27 \pm 0.29(0.07-0.36), 28$ & $\dagger$ & & \\
\hline
\end{tabular}

Data were shown in mean $\pm S D$; $I Q R$, interquartile range; extremum.

†, Not measured; and P-values were computed based on two-sample t-test. BF, Bayes factor; GDS, global deficit score.

resting-state scan, subjects were instructed to stay awake and to remain as still as possible while keeping their eyes closed. Restingstate fMRI images were acquired via an echo-planar imaging sequence (echo time $=25 \mathrm{~ms}$, repetition time $=2,000 \mathrm{~ms}$, flip angle $=75^{\circ}$, field of view $=224 \times 224 \mathrm{~mm}$, axial slices $=34$, slice thickness $=4 \mathrm{~mm}$, and voxel size $=1.0 \times 1.0 \times 1.0 \mathrm{~mm}$, 200 time points). T1-weighted structural images were obtained on a sagittal orientation employing a magnetization-prepared rapid gradient-echo sequence (echo time $=3.8 \mathrm{~ms}$, repetition time $=1,900 \mathrm{~ms}$, flip angle $=8^{\circ}$, field of view $=240 \times 240 \mathrm{~mm}$, 170 slices per slab, voxel size $=1.0 \times 1.0 \times 1.0 \mathrm{~mm}$, and matrix $=256 \times 256)$.

\section{MRI Data Analysis}

The VBM analysis was performed using Statistical Parametric Mapping (SPM12) with the VBM8 toolbox. The raw data were first checked for the scanner artifacts and anatomical abnormalities. Subsequently, the whole-brain T1-weighted images were segmented into gray matter, white matter, and cerebrospinal fluid images, and were normalized to adjust for differences in volume. Finally, all segmented images were smoothened with an $8 \mathrm{~mm}$ full width at a half-maximum (FWHM) Gaussian kernel standard to increase the signal-to-noise ratio. Significance was identified using a voxel-level threshold of $P<0.001$ and a cluster-level threshold of $P<0.05$ with family wise-error (FWE) correction for multiple comparisons.

The resting-state $\mathrm{fMRI}$ data were preprocessed using the Statistical Parametric Mapping (SPM12) and the Resting-State fMRI Data Analysis Toolkit (REST, http://www.restfmri.net). After discarding the first 10 volumes, the remaining fMRI images were realigned, and slice-timing was corrected. The images were then co-registered to individual T1-weighted images and spatially normalized using the Montréal Neurological Institute (MNI) template. We removed subjects who had head motion exceeding $3.0 \mathrm{~mm}$ of maximal translation (in any direction of $\mathrm{x}, \mathrm{y}$, or $\mathrm{z}$ ) or $3.0^{\circ}$ of maximal rotation during the course of scanning. The normalized images were re-sampled to an isotropic voxel size of $3.0 \times 3.0 \times 3.0 \mathrm{~mm}$ and smoothened using an FWHM Gaussian kernel of $6 \mathrm{~mm}$ before they were subjected to the removal of the linear drift. Six head motion parameters, and ventricle and white matter signals were regressed out before temporal filtering $(0.01-0.08 \mathrm{~Hz})$.

The clusters identified from the VBM analysis were defined as regions of interest (ROIs) and served as the seeds in the seedbased functional connectivity analysis. For each participant, the time courses of voxels in each ROI were extracted and averaged across voxels. Pearson correlation was computed between the seed time series and time series of other voxels in the brain, and the correlation coefficients were transformed into Fisher's Zscores. Group differences were compared with controlling age as a covariate. Statistical significance was identified using a voxellevel threshold of $P<0.001$ and a cluster-level threshold of $P<$ 0.05 with FWE correction for multiple comparisons.

The ALFF value of each voxel was calculated by averaging the square root of the power spectrum with $0.01-0.08 \mathrm{~Hz}$. The regional homogeneity value of each voxel was estimated by calculating Kendall's coefficient of concordance of the given voxel along with its adjacent 26 voxels. Group comparisons of ALFF and regional homogeneity values were performed using the twosample $t$-test with age controlled as a covariate, and significance was identified using a voxel-level threshold of $P<0.001$ and a cluster-level threshold of $P<0.05$ with FWE correction for multiple comparisons.

In addition to the standard framework of frequentist statistics featuring $P$-value null-hypothesis significance testing, we calculated the Bayes factors $\left(\mathrm{BF}_{+0}\right)$ based on $t$-values and sample sizes using the BayesFactor package in $\mathrm{R}$ (https://cran. r-project.org/web/packages/BayesFactor/index.html) (25). $\mathrm{BF}_{+0}$ $\geq 3$ provides evidence for the alternative hypothesis; $\mathrm{BF}_{+0} \leq$ $1 / 3$ provides evidence for the null hypothesis; $1 / 3 \leq \mathrm{BF}_{+0} \leq 3$ indicate that there is insufficient evidence to draw a conclusion for or against either hypothesis (26).

\section{RESULTS}

As shown in Figure 1 and Table 2, the HIV+ HoM group had significantly higher GMV mainly in the 


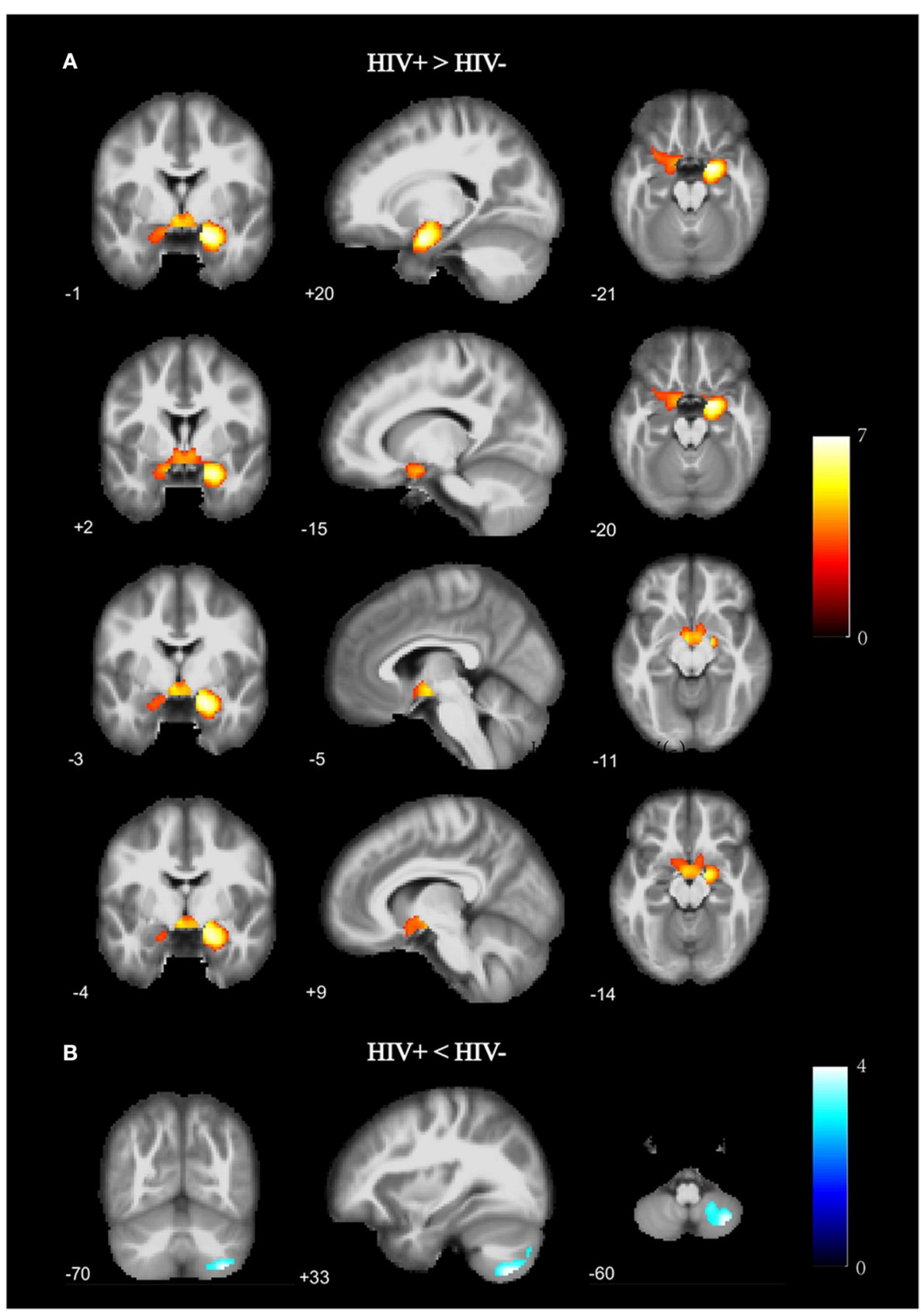

FIGURE 1 | Gray matter volume (GMV) differences between HIV+ HoM and HIV- HoM based on voxel-based morphometry (VBM) analysis. (A) Brain regions with significantly increased gray matter in the HIV $+\mathrm{HoM}$ group (voxel-level uncorrected $P<0.001$, cluster-level FWE-corrected $P<0.05$ ). (B) Brain regions with significantly decreased gray matter in the HIV+ HoM group (voxel-level uncorrected $P<0.001$, cluster-level FWE-corrected $P<0.05$ ). The color bars indicate $T$-statistics.

bilateral parahippocampal gyrus (ParaHippocampal_R and ParaHippocampal_L, brain labels were based on the AAL3 atlas) and amygdala (Amygdala_L and Amygdala_R) compared with HIV- HoM controls after controlling for age but reduced GMV were observed in the right inferior cerebellum (Cerebelum_8_R) (FWE corrected, voxel-level $P<0.001$, cluster-level $P<0.05)$.
The cluster with increased GMV in the parahippocampal gyrus and amygdala found in the VBM analysis was then used as the seed in the seed-based functional connectivity analysis of rs-fMRI data. No significant group difference was found using the strict threshold (voxel-level $P<0.001$, cluster-level $P<0.05$ with FWE correction). With a less strict threshold (voxel-level uncorrected $P<0.005$, cluster size $>50$ ), the 
TABLE 2 | Gray matter volume differences between HIV+ HoM and HIV- HoM.

\begin{tabular}{|c|c|c|c|c|c|c|c|}
\hline \multirow[t]{2}{*}{ Region } & \multicolumn{3}{|c|}{ Coordinates } & \multirow[t]{2}{*}{ Cluster Size } & \multirow[t]{2}{*}{$T$-value } & \multirow[t]{2}{*}{$P$-value } & \multirow[t]{2}{*}{ BF } \\
\hline & $\mathbf{x}$ & $\mathbf{y}$ & $\mathbf{z}$ & & & & \\
\hline \multicolumn{8}{|l|}{$\mathrm{HIV}_{+}>\mathrm{HIV}_{-}$} \\
\hline ParaHippocampal_R & 20 & -1 & -21 & 3,614 & 7.281 & $<0.0000$ & $39,130,564$ \\
\hline Amygdala_L & -5 & -3 & -11 & 3,614 & 5.293 & $<0.0000$ & 13,729 \\
\hline Amygdala_R & 9 & -4 & -14 & 3,614 & 4.659 & $<0.0000$ & 1,429 \\
\hline ParaHippocampal_L & -15 & 2 & -20 & 3,614 & 4.613 & $<0.0000$ & 1,220 \\
\hline Cerebelum_8_L & -17 & -69 & -38 & 95 & 4.760 & $<0.0000$ & 2,024 \\
\hline Putamen_L & -30 & -9 & 6 & 62 & 4.375 & $<0.0000$ & 550 \\
\hline \multicolumn{8}{|l|}{$\mathrm{HIV}_{+}<\mathrm{HIV}-$} \\
\hline Cerebelum_8_R & 33 & -70 & -60 & 874 & -4.296 & 0.0001 & 424 \\
\hline Calcarine_L & 0 & -78 & 7 & 715 & -4.121 & $<0.0000$ & 242 \\
\hline Cerebelum_Crus2_L & -32 & -83 & -41 & 337 & -4.506 & $<0.0000$ & 849 \\
\hline Hippocampus_R & 27 & -27 & -5 & 206 & -5.065 & $<0.0000$ & 5,969 \\
\hline Thalamus_R & 12 & -29 & 12 & 145 & -4.061 & 0.0001 & 201 \\
\hline Cerebelum_6_R & 8 & -65 & -15 & 67 & -3.548 & 0.0007 & 44 \\
\hline
\end{tabular}

BF, Bayes factor. Brain regions at a voxel-level threshold of $P<0.001$ and a cluster-level threshold of $P<0.05$ with FWE correction for multiple comparisons were highlighted.

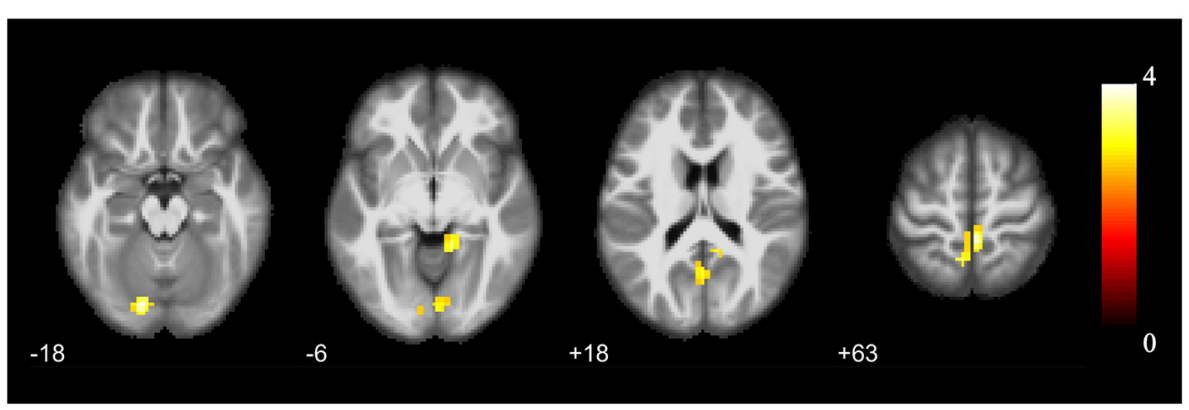

FIGURE 2 | Brain regions with increased functional connectivity to the cluster with increased GMV (parahippocampal gyrus and amygdala) in HIV+ HoM compared with HIV- HoM (voxel-level uncorrected $P<0.005$, cluster size $>50)$. The color bars indicate $T$-statistics.

TABLE 3 | Functional connectivity differences between HIV+ HoM and HIV- HoM.

$\begin{array}{ll}\text { Region } & \frac{\text { Coordinates Cluster Size } T \text {-value } P \text {-value BF }}{\text { x y z }}\end{array}$

$\mathrm{HIV}+>\mathrm{HIV}-$

\begin{tabular}{lccccccc} 
Cerebelum_Crus1_L & -15 & -84 & -18 & 96 & 4.022 & 0.0001 & 178 \\
Lingual_R & 12 & -45 & -6 & 92 & 3.619 & 0.0005 & 53 \\
Precuneus_L & 0 & -63 & 18 & 57 & 3.198 & 0.0020 & 17 \\
\hline
\end{tabular}

BF, Bayes factor. Corrected for multiple comparisons (voxel-level uncorrected $P<0.005$, cluster size > 50).

functional connectivity strength of the cluster with the left superior cerebellum (Cerebelum_Crus1_L), right lingual gyrus (Lingual_R), and left precuneus (Precuneus_L) was decreased in the HIV+ HoM group compared with the HIV- HoM controls as shown in Figure 2 and Table 3.

No significant group difference was found in ALFF and regional homogeneity values using the strict threshold (voxel-level $P<0.001$, cluster-level $P<0.05$ with FWE correction). With a less strict threshold (voxel-level uncorrected $P<0.005$, cluster-level $P<0.05$ ), the HIV + HoM exhibited an increase in ALFF in the right fusiform gyrus (Fusiform_R) and left parahippocampal gyrus (ParaHippocampal_L) as shown in Figure 3A and Table 4. With a less strict threshold (voxel-level uncorrected $P<0.005$, cluster size $>50$ ), the HIV+ HoM exhibited a decrease in regional homogeneity in the right anterior cingulate and paracingulate gyri (Cingulate_Ant_R), and left superior cerebellum (Cerebelum_Crus1_L) as shown in Figure 3B and Table 5.

\section{DISCUSSION}

The current study investigated the impact of HIV on brain structure and function in a cohort of adults with the same sexual orientation. We found that, in homosexual males, HIV infection showed greater GMV of limbic structures (parahippocampal gyrus and amygdala), and increased functional connectivity of these limbic structures with cerebellum, and brain regions 


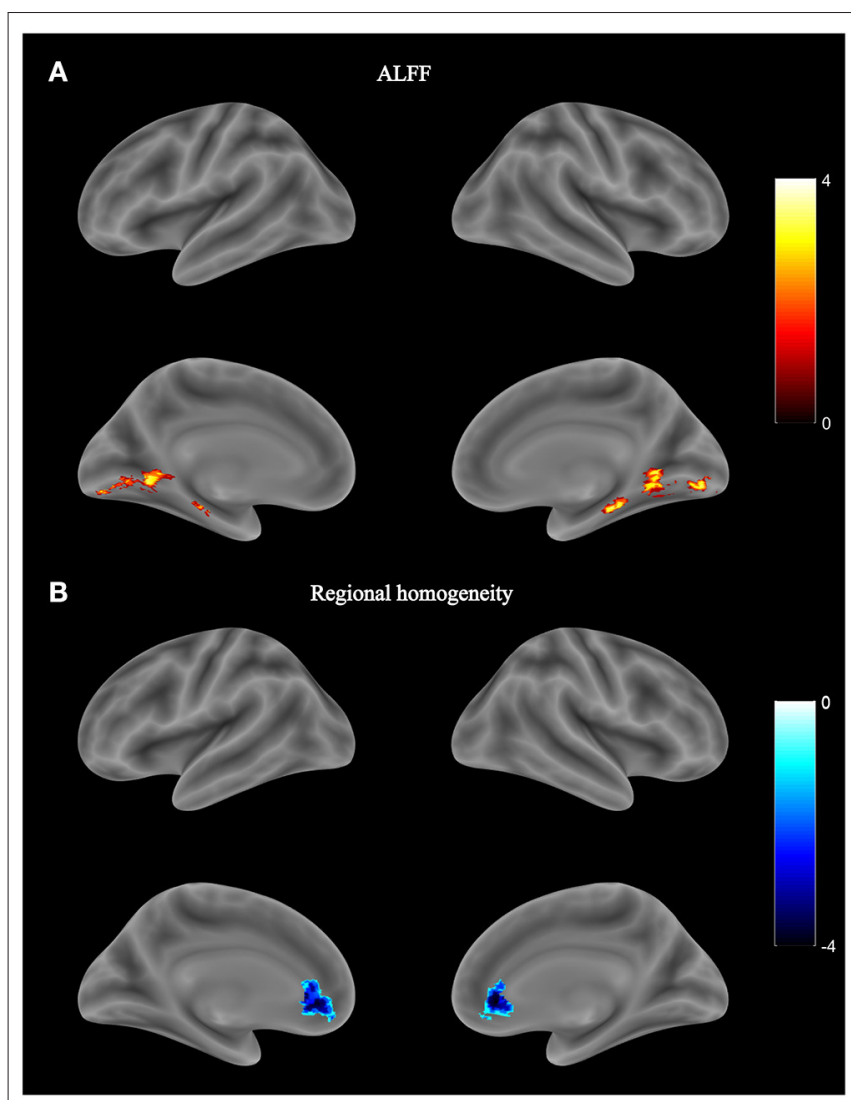

FIGURE 3 | Local functional abnormality in HIV+ HoM compared with HIVHoM. (A) Brain regions with increased amplitude of low-frequency fluctuation (ALFF) values in the HIV+ HoM group (voxel-level uncorrected $P<0.005$, cluster-level FWE-corrected $P<0.05$ ). (B) Brain regions with decreased regional homogeneity in the HIV + HoM group (voxel-level uncorrected $P<$ 0.005 , cluster size $>50$ ). The color bars indicate $T$-statistics.

TABLE 4 | Amplitude of low-frequency fluctuation (ALFF) differences between $\mathrm{HIV}+\mathrm{HoM}$ and HIV- HoM.

\begin{tabular}{|c|c|c|c|c|c|c|}
\hline \multirow[t]{2}{*}{ Region } & \multicolumn{3}{|c|}{ Coordinates } & \multirow[t]{2}{*}{ Cluster Size } & \multirow[t]{2}{*}{$T$-value } & \multirow[t]{2}{*}{$P$-value BF } \\
\hline & $\mathbf{x}$ & $y$ & $z$ & & & \\
\hline
\end{tabular}

$\mathrm{HIV}+>\mathrm{HIV}_{-}$

$\begin{array}{lccccccc}\text { Fusiform_R } & 24 & -33 & -21 & 147 & 3.814 & 0.0003 & 94 \\ \text { ParaHippocampal_L } & -21 & -27 & -18 & 210 & 3.778 & 0.0003 & 85\end{array}$

$B F$, Bayes factor. Corrected for multiple comparisons (voxel-level uncorrected $P<0.005$, cluster-level FWE-corrected $P<0.05$ ).

associated with the visual network (middle occipital gyrus and lingual gyrus). These results provided evidence of brain abnormalities in people living with HIV infection.

Our major finding was the structural and functional dysfunction of the parahippocampal gyrus in the HIV+ HoM. Several previous studies have repeatedly reported the impact of $\mathrm{HIV}$ infection on the parahippocampal gyrus. A previous study comparing the HIV+ older adults with age-matched uninfected controls showed reduced GMV in the parahippocampal gyrus
TABLE 5 | Regional homogeneity differences between HIV+ HoM and HIV- HoM.

\begin{tabular}{lll}
\hline Region & \multicolumn{2}{l}{ Coordinates } \\
\cline { 1 - 3 } & Cluster Size $T$-value $P$-value BF \\
\hline
\end{tabular}

$\mathrm{HIV}_{+}<\mathrm{HIV}-$

\begin{tabular}{lccccccc} 
Cingulate_Ant_R & 3 & 33 & 0 & 195 & -4.328 & 0.0000 & 471 \\
Cerebelum_Crus1_L & -18 & -72 & -30 & 56 & -3.678 & 0.0004 & 63 \\
\hline
\end{tabular}

$B F$, Bayes factor. Corrected for multiple comparisons (voxel-level uncorrected $P<0.005$, cluster size $>50)$.

(27). Another study about people living with HIV infection also found brain atrophy in the hippocampus/parahippocampal gyrus (28). In contrast to these previous findings, our main finding was that the HIV+ HoM group had a greater GMV in the bilateral parahippocampal gyrus compared with controls. Compared to the relative abundance of literature elucidating the structural atrophy to inflammation and immune function (2931), the mechanisms of pathogenesis, such as hypertrophy of brain structures have not been well-understood. As we studied a relatively young cohort compared to previous studies, we speculated that the increased GMV of the parahippocampal gyrus may have been related to the early compensation of brain functions due to the brain parenchymal lesions induced by HIV infection. This speculation was further supported by the enhanced regional function in this region as indicated by the increased ALFF. The parahippocampal gyrus has been associated with many cognitive functions, especially in episodic memory and visuospatial processing (32). Our study also reported increased functional connectivity between the parahippocampal gyrus and the precuneus, which participates in memory-related activities and is closely connected to the hippocampus (33). Thus, the abnormalities we reported in the parahippocampal gyrus may affect memory, providing supporting evidence to the memory dysfunction associated with HIV infection $(34,35)$.

In addition to the parahippocampal gyrus, the amygdala also showed increased GMV in this study. As an important nucleus that can affect mood, emotion, learning, and memory function, the GMV changes in the amygdala were often involved in neurological or psychiatric diseases, such as Asperger syndrome (36), anxiety (37), and advanced depression (38). A neuroimaging study involving HIV + individuals exposed to early life stress showed that high levels of stress and HIV could interact to increase the amygdala volume and may result in neurocognitive dysfunction in $\mathrm{HIV}+$ individuals (39). In terms of sexual orientation, the amygdala is an important component of the core neural pathway of male sexual arousal and is responsible for emotional response regulation (40). The activation of the amygdala in HoM was greater than heterosexual males in response to specific visual stimuli (41). It is possible that the sexual orientation and HIV could also interact to increase the amygdala volume. However, the potential interaction between male homosexual orientation and HIV infection remains to be further investigated. In addition, the cingulate gyrus, which is reciprocally connected with amygdala, showed decreased regional homogeneity in the HIV+ HoM of our study. 
We found decreased GMV in the cerebellum in the HIV+ HoM, which is consistent with previous studies $(42,43)$. The function of left superior cerebellum was also disrupted as indicated by the decreased regional homogeneity in the HIV + HoM. Furthermore, we found increased functional connectivity between the left superior cerebellum and the cortical areas with greater GMV (mainly the parahippocampal gyrus) in the HIV+ HoM group comparing to the HIVHoM. This is in accordance with the previous studies indicating important functional interactions between the cerebellum and the hippocampus formation. For instance, functional connectivity between the left hippocampus and the bilateral cerebellum was increased in HIV+ individuals, which was associated with the spatio-temporal prediction of movements in the memory formation (44). In a study of cerebellar functional connectivity in HIV+ male individuals, decreased functional connectivity between the right lobule VI and the left hippocampus was considered as the brain mechanism underlying the impairment of spatial and temporal processing function (45). As the parahippocampal gyrus is the main surrounding structure of the hippocampus and plays an important role in memory encoding and retrieval (46), the increased functional connectivity between the parahippocampal gyrus and cerebellum found in the current study might similarly indicate the behavioral impairments in memory functions.

The HIV + HoM group also exhibited functional abnormality in the brain regions involved in the visual network. The lingual gyrus plays an important role in visual attention and visual judgment (46). As discussed previously (41), the HoM showed greater activation of the amygdala when processing specific visual stimuli. The increased functional connectivity we found between the visual areas and brain structures with increased GMV including the amygdala in the HIV+ HoM group might suggest a certain relationship between the HIV infection and HoM. We also found a local functional abnormality in the visual network in the HIV+ HoM, demonstrated by the increased ALFF values in the fusiform gyrus involved in visual processing. As little evidence has been reported in previous studies, the relationship between visual cortex dysfunction and HIV infection needs to be further studied.

There were several limitations in this study. First, the grouping of sexual orientation was based on the self-identification of participants, lacking a measurement basis such as the Kinsey scale (47). We might have included participants who were "homosexual but with incidental or occasional heterosexual tendencies," which is separated from "exclusive homosexual" on the Kinsey scale. The current findings need to be further verified using exclusive homosexual individuals. Second, the clinical information of enrolled individuals was incomplete, including some important confounding factors, such as the medication regimen of $\mathrm{HIV}+$ patients. And although individuals having a history of alcohol or drug abuse were excluded in our study, these conditions were obtained via self-report and concealment of illicit drug use could not be ruled out. Thus, we could not completely eliminate the influence of such confounding factors on the results. Third, the present study did not conduct a full set of neurocognitive tests in all HIV+ patients, and the explanation for the positive results of structural and functional MRI was mainly dependent on the reports from other scholars. Future research involving a specific neurocognitive performance may help address some of these questions.

To our best knowledge, this is the first study to strictly control sexual orientation while investigating the HIV-related alteration in the brain structure and function. We found significant structural abnormalities of the limbic system and cerebellum, and changes in functional connectivity and activity in the brain regions related to memory and visual function in the HIV + HoM compared with the HIV- HoM controls. Further studies are needed to expand the sample size, acquire relevant clinical information, and group strictly according to confounding factors, and employ multimodal imaging methods, so as to improve our understanding of the pathophysiological mechanism of HIV infection in the brain.

\section{DATA AVAILABILITY STATEMENT}

The original contributions presented in the study are included in the article/supplementary material, further inquiries can be directed to the corresponding author/s.

\section{ETHICS STATEMENT}

The studies involving human participants were reviewed and approved by the Ethics Committee of the Shanghai Public Health Clinical Center. The patients/participants provided their written informed consent to participate in this study.

\section{AUTHOR CONTRIBUTIONS}

QM and XS: study design, participants recruitment, data collection, and data analysis. GC: data collection and data analysis. FS and FL: data analysis. HZ: participants recruitment. YS and D-CC: study conceptualization, project administration, and data analysis. All authors contributed to the article and approved the submitted version.

\section{FUNDING}

This work was supported by the Foundations of Shanghai Municipal Population and Family Planning Commission (201840146) and Science and Technology Commission of Shanghai Municipality (19411965800). 


\section{REFERENCES}

1. Davis LE, Hjelle BL, Miller VE, Palmer DL, Llewellyn AL, Merlin TL, et al. Early viral brain invasion in iatrogenic human immunodeficiency virus infection. Neurology. (1992) 42:1736-9. doi: 10.1212/WNL.42.9.1736

2. Clifford DB, Ances BM. HIV-associated neurocognitive disorder. Lancet Infect Dis. (2013) 13:976-86. doi: 10.1016/S1473-3099(13)70269-X

3. Campbell LM, Fennema-Notestine C, Saloner R, Hussain M, Chen A, Franklin D, et al. Use of neuroimaging to inform optimal neurocognitive criteria for detecting HIV-associated brain abnormalities. J Int Neuropsychol Soc. (2020) 26:147-62. doi: 10.1017/S1355617719000985

4. Boerwinkle AH, Meeker KL, Luckett P, Ances BM. Neuroimaging the neuropathogenesis of HIV. Curr HIV/AIDS Rep. (2021) 18:221-8. doi: 10.1007/s11904-021-00548-Z

5. Kallianpur KJ, Gerschenson M, Mitchell BI, LiButti DE, Umaki TM, Ndhlovu LC, et al. Oxidative mitochondrial DNA damage in peripheral blood mononuclear cells is associated with reduced volumes of hippocampus and subcortical gray matter in chronically HIV-infected patients. Mitochondrion. (2016) 28:8-15. doi: 10.1016/j.mito.2016.02.006

6. Sanford R, Fernandez Cruz AL, Scott SC, Mayo NE, Fellows LK, Ances BM, et al. Regionally specific brain volumetric and cortical thickness changes in HIV-infected patients in the HAART era. J Acquir Immune Defic Syndr. (2017) 74:563-70. doi: 10.1097/QAI.0000000000001294

7. Li J, Gao L, Wen Z, Zhang J, Wang P, Tu N, et al. Structural covariance of gray matter volume in HIV vertically infected adolescents. Sci Rep. (2018) 8:1182. doi: 10.1038/s41598-018-19290-5

8. Gawron N, Choiński M, Szymańska-Kotwica B, Pluta A, Sobańska M, Egbert AR, et al. Effects of age, HIV, and HIV-associated clinical factors on neuropsychological functioning and brain regional volume in HIV+ patients on effective treatment. I Neurovirol. (2019) 25:921. doi: 10.1007/s13365-018-0679-4

9. O'Connor EE, Zeffiro TA, Zeffiro TA. Brain structural changes following HIV infection: meta-analysis. Am J Neuroradiol. (2018) 39:54-62. doi: 10.3174/ajnr.A5432

10. Van den Hof M, Ter Haar AM, Caan MWA, Spijker R, van der Lee JH, Pajkrt D. Brain structure of perinatally HIV-infected patients on longterm treatment: a systematic review. Neurol Clin Pract. (2019) 9:43342. doi: 10.1212/CPJ.0000000000000637

11. Thomas JB, Brier MR, Snyder AZ, Vaida FF, Ances BM. Pathways to neurodegeneration: effects of HIV and aging on resting-state functional connectivity. Neurology. (2013) 80:118693. doi: 10.1212/WNL.0b013e318288792b

12. Chaganti JR, Heinecke A, Gates TM, Moffat KJ, Brew BJ. Functional connectivity in virally suppressed patients with HIV-associated neurocognitive disorder: a resting-state analysis. Am J Neuroradiol. (2017) 38:1623-9. doi: 10.3174/ajnr.A5246

13. Mackiewicz MM, Overk C, Achim CL, Masliah E. Pathogenesis of age-related HIV neurodegeneration. J Neurovirol. (2019) 25:622-33. doi: 10.1007/s13365-019-00728-Z

14. Seider TR, Gongvatana A, Woods AJ, Chen H, Porges EC, Cummings T, et al. Age exacerbates HIV-associated white matter abnormalities. J Neurovirol. (2016) 22:201-12. doi: 10.1007/s13365-015-0386-3

15. Ortega M, Brier MR, Ances BM. Effects of HIV and combination antiretroviral therapy on cortico-striatal functional connectivity. Aids. (2015) 29:70312. doi: $10.1097 / \mathrm{QAD} .0000000000000611$

16. Young AC, Yiannoutsos CT, Hegde M, Lee E, Peterson J, Walter R, et al. Cerebral metabolite changes prior to and after antiretroviral therapy in primary HIV infection. Neurology. (2014) 83:1592-600. doi: 10.1212/WNL.0000000000000932

17. Witelson SF, Kigar DL, Scamvougeras A, Kideckel DM, Buck B, Stanchev PL, et al. Corpus callosum anatomy in right-handed homosexual and heterosexual men. Arch Sex Behav. (2008) 37:857-63. doi: 10.1007/s10508-007-92 76-y

18. Abé C, Johansson E, Allzén E, Savic I. Sexual orientation related differences in cortical thickness in male individuals. PLoS ONE. (2014) 9:e114721. doi: 10.1371/journal.pone.0114721

19. Manzouri A, Savic I. Cerebral sex dimorphism and sexual orientation. Hum Brain Mapp. (2018) 39:1175-86. doi: 10.1002/hbm.23908
20. Savic I, Lindström P. PET and MRI show differences in cerebral asymmetry and functional connectivity between homo- and heterosexual subjects. Proc Natl Acad Sci USA. (2008) 105:9403-8. doi: 10.1073/pnas.0801566105

21. Hu S, Xu D, Peterson BS, Wang Q, He X, Hu J, et al. Association of cerebral networks in resting state with sexual preference of homosexual men: a study of regional homogeneity and functional connectivity. PLoS ONE. (2013) 8:e59426. doi: 10.1371/journal.pone.0059426

22. Hu S, Xu D, Peterson BS, Wang Q, Lai J, Hu J, et al. Differing default mode network activities in men with homosexual or heterosexual preferences. J Sex Med. (2014) 11:2474-84. doi: 10.1111/jsm.12639

23. UNAIDS. UNAIDS Data 2019. Available online at: https://www.unaids. org/en/resources/documents/2019/2019-UNAIDS-data (accessed December 4, 2019).

24. Carey CL, Woods SP, Gonzalez R, Conover E, Marcotte TD, Grant I, et al. Predictive validity of global deficit scores in detecting neuropsychological impairment in HIV infection. J Clin Exp Neuropsychol. (2004) 26:30719. doi: 10.1080/13803390490510031

25. Morey RD, Rouder JN, Jamil T, Urbanek S, Forner K, Ly A. BayesFactor: Computation of Bayes Factors for Common Designs. Netherlands (2015).

26. Keysers C, Gazzola V, Wagenmakers EJ. Using Bayes factor hypothesis testing in neuroscience to establish evidence of absence. Nat Neurosci. (2020) 23:78899. doi: 10.1038/s41593-020-0660-4

27. Wilson TW, Heinrichs-Graham E, Becker KM, Aloi J, Robertson KR, Sandkovsky U, et al. Multimodal neuroimaging evidence of alterations in cortical structure and function in HIV-infected older adults. Hum Brain Mapp. (2015) 36:897-910. doi: 10.1002/hbm.22674

28. Liu D, Zhao C, Wang W, Wang Y, Li R, Sun J, et al. Altered gray matter volume and functional connectivity in human immunodeficiency virus-infected adults. Front Neurosci. (2020) 14:601063. doi: 10.3389/fnins.2020.601063

29. Lindl KA, Marks DR, Kolson DL, Jordan-Sciutto KL. HIV-associated neurocognitive disorder: pathogenesis and therapeutic opportunities. $J$ Neuroimmune Pharmacol. (2010) 5:294-309. doi: 10.1007/s11481-010-9205-Z

30. Saloner R, Cysique LA. HIV-associated neurocognitive disorders: a global perspective. J Int Neuropsychol Soc. (2017) 23:8609. doi: $10.1017 / \mathrm{S} 1355617717001102$

31. Sénécal V, Barat $C$, Tremblay MJ. The delicate balance between neurotoxicity and neuroprotection in the context of HIV-1 infection. Glia. (2021) 69:25580. doi: 10.1002/glia.23904

32. Aminoff EM, Kveraga K, Bar M. The role of the parahippocampal cortex in cognition. Trends Cogn Sci. (2013) 17:379-90. doi: 10.1016/j.tics.2013.06.009

33. Ren Y, Nguyen VT, Sonkusare S, Lv J, Pang T, Guo L, et al. Effective connectivity of the anterior hippocampus predicts recollection confidence during natural memory retrieval. Nat Commun. (2018) 9:4875. doi: 10.1038/s41467-018-07325-4

34. Rubin LH, Meyer VJ, R JC, Sundermann EE, Wu M, Weber KM, et al. Prefrontal cortical volume loss is associated with stress-related deficits in verbal learning and memory in HIV-infected women. Neurobiol Dis. (2016) 92(Pt B):166-74. doi: 10.1016/j.nbd.2015.09.010

35. Cohen RA, Siegel S, Gullett JM, Porges E, Woods AJ, Huang H, et al. Neural response to working memory demand predicts neurocognitive deficits in HIV. J Neurovirol. (2018) 24:291-304. doi: 10.1007/s13365-017-0607-z

36. Murphy CM, Deeley Q, Daly EM, Ecker C, O’Brien FM, Hallahan B, et al. Anatomy and aging of the amygdala and hippocampus in autism spectrum disorder: an in vivo magnetic resonance imaging study of Asperger syndrome. Autism Res. (2012) 5:3-12. doi: 10.1002/aur.227

37. Hayano F, Nakamura M, Asami T, Uehara K, Yoshida T, Roppongi $\mathrm{T}$, et al. Smaller amygdala is associated with anxiety in patients with panic disorder. Psychiatry Clin Neurosci. (2009) 63:266-76. doi: 10.1111/j.1440-1819.2009.01960.x

38. Burke J, McQuoid DR, Payne ME, Steffens DC, Krishnan RR, Taylor WD. Amygdala volume in late-life depression: relationship with age of onset. Am J Geriatr Psychiatry. (2011) 19:771-6. doi: 10.1097/JGP.0b013e318211069a

39. Clark US, Cohen RA, Sweet LH, Gongvatana A, Devlin KN, Hana GN, et al. Effects of HIV and early life stress on amygdala morphometry and neurocognitive function. J Int Neuropsychol Soc. (2012) 18:65768. doi: 10.1017/S1355617712000434

40. Kühn S, Gallinat J. A quantitative meta-analysis on cue-induced male sexual arousal. J Sex Med. (2011) 8:2269-75. doi: 10.1111/j.1743-6109.2011.02322.x 
41. Safron A, Barch B, Bailey JM, Gitelman DR, Parrish TB, Reber PJ. Neural correlates of sexual arousal in homosexual and heterosexual men. Behav Neurosci. (2007) 121:237-48. doi: 10.1037/0735-7044.121.2.237

42. Sanford R, Ances BM, Meyerhoff DJ, Price RW, Fuchs D, Zetterberg H, et al. Longitudinal trajectories of brain volume and cortical thickness in treated and untreated primary human immunodeficiency virus infection. Clin Infect Dis. (2018) 67:1697-704. doi: 10.1093/cid/ciy362

43. Yu X, Gao L, Wang H, Yin Z, Fang J, Chen J, et al. Neuroanatomical changes underlying vertical HIV infection in adolescents. Front Immunol. (2019) 10:814. doi: 10.3389/fimmu.2019.00814

44. Onuki Y, Van Someren EJ, De Zeeuw CI, Van der Werf YD. Hippocampalcerebellar interaction during spatio-temporal prediction. Cereb Cortex. (2015) 25:313-21. doi: 10.1093/cercor/bht221

45. Wang H, Li R, Zhou Y, Wang Y, Cui J, Nguchu BA, et al. Altered cerebro-cerebellum resting-state functional connectivity in $\mathrm{HIV}$-infected male patients. J Neurovirol. (2018) 24:587-96. doi: 10.1007/s13365-018-0649-x

46. Lee HW, Hong SB, Seo DW, Tae WS, Hong SC. Mapping of functional organization in human visual cortex: electrical cortical stimulation. Neurology. (2000) 54:849-54. doi: 10.1212/WNL.54.4.849

47. Kinsey AC, Pomeroy WR, Martin CE. Sexual behavior in the human male 1948. Am J Public Health. (2003) 93:894-8. doi: 10.2105/AJPH.9 3.6 .894
48. Manzouri A, Savic I. Multimodal MRI suggests that male homosexuality may be linked to cerebral midline structures. PLoS ONE. (2018) 13:e203189. doi: 10.1371/journal.pone.0203189

Conflict of Interest: The authors declare that the research was conducted in the absence of any commercial or financial relationships that could be construed as a potential conflict of interest.

Publisher's Note: All claims expressed in this article are solely those of the authors and do not necessarily represent those of their affiliated organizations, or those of the publisher, the editors and the reviewers. Any product that may be evaluated in this article, or claim that may be made by its manufacturer, is not guaranteed or endorsed by the publisher.

Copyright (c) 2022 Ma, Shi, Chen, Song, Liu, Zheng, Shi and Cai. This is an openaccess article distributed under the terms of the Creative Commons Attribution License (CC BY). The use, distribution or reproduction in other forums is permitted provided the original author(s) and the copyright owner(s) are credited and that the original publication in this journal is cited, in accordance with accepted academic practice. No use, distribution or reproduction is permitted which does not comply with these terms. 